\title{
Asociación entre el estado de ánimo negativo, el consumo de sustancias psicoactivas y el bullying en adolescentes escolarizados
}

\section{Association between negative mood states, psychoactive substances consumption and bullying in school-aged adolescents}

\author{
Ainara Díaz-Geada*, Albert Espelt**,***,****, Marina Bosque-Prous*****, \\ Núria Obradors-Rial**, Ester Teixidó-Compañó**, Francisco CaAmaño Isorna*,****. \\ * Área de Medicina Preventiva y Salud Pública. Universidade de Santiago de Compostela. ** Facultat de Ciències de la Salut de \\ Manresa. Universitat de Vic Universitat Central de Catalunya (UVicUCC). Av. Universitària. *** Departament de Psicobiologia \\ i Metodologia en Ciències de la Salut. Universitat Autònoma de Barcelona (UAB). **** Centro de Investigación Biomédica en \\ Red de Epidemiología y Salud Pública (CIBERESP). ***** Facultat de Ciències de la Salut. Universitat Oberta de Catalunya.
}

\section{Resumen}

Antecedentes: los problemas de salud mental durante la adolescencia suponen un aumento de la morbimortalidad y la discapacidad. Se pretende testar la hipótesis de que el bullying y el consumo de sustancias psicoactivas están asociados al estado de ánimo negativo.

Métodos: estudio transversal entre estudiantes de Educación Secundaria Obligatoria (ESO) $(n=238)$ de los institutos de Burela (Lugo). El "estado de ánimo negativo" se definió a partir de los siguientes ítems: sentirse cansado/a, triste, desplazado/a, aburrido/a, desesperanzado/a, nervioso/a o insomne. Como variables independientes se consideraron: el binge drinking, el haber fumado alguna vez tabaco o cannabis, así como sus correspondientes percepciones de peligrosidad. Además, se midió la variable bullying. Se estimaron modelos de regresión de Poisson con varianza robusta y se obtuvieron Razones de Prevalencia (RP).

Resultados: el 10,5\% [IC95\% (7,2-15,2)] de la población encuestada presentaba estado de ánimo negativo. La nula o baja percepción de peligrosidad para el cannabis $[\mathrm{RP}=2,6(1,2-5,5)]$, haber probado alguna vez esta sustancia adictiva $[\mathrm{RP}=3,1 \quad(1,1-8,9)]$ y haber sufrido bullying $[\mathrm{RP}=4,8(2,4-9,6)]$ se asociaban al estado de ánimo negativo. Conclusiones: sería recomendable crear intervenciones para la mejora de la salud mental durante la adolescencia que tengan en cuenta el consumo de sustancias adictivas y el hecho de haber sufrido bullying. Palabras clave: estado de ánimo negativo, consumo de sustancias, adolescentes, bullying.

\begin{abstract}
Objective: mental health problems during adolescence lead to increased morbidity and mortality. We intend to test the hypothesis that bullying and addictive substance use is related to negative mood states.

Methods: We carried out a cross-sectional study among high school students in Burela (Northern Spain) $(n=238)$. "Negative mood state" was defined as experiencing the following: feeling tired, sad, out of place, bored, hopeless, nervous or lacking sleep. Independent variables were binge drinking, having smoked tobacco or cannabis, and the corresponding perceived risk of using them. The variable bullying was also measured. Poisson regression models with robust variance were estimated, and Prevalence Ratios were obtained.

Results: $10.5 \%$ [CI 95\% (7.2-15.2)] of the students reported suffering negative mood states. Students declaring low perceived risk of cannabis use $[\mathrm{PR}=2.6(1.2-5.5)]$, having tried this addictive substance at some point $[\mathrm{PR}=3.1(1.1-8.9)]$ and having suffered bullying $[\mathrm{PR}=$ 4.8 (2.4-9.6)] increased the risk of experiencing negative mood states. Conclusion: It would be advisable to design and implement interventions aimed at improving mental health during adolescence which account for the use of addictive substances and being a victim of bullying.

Key Words: negative mood states, substance use, adolescents, bullying.
\end{abstract}


$\mathrm{D}$ urante la adolescencia, procesos emocionales prolongados en el tiempo como la desesperanza, la tristeza, la soledad o el nerviosismo definen el estado de ánimo negativo, el cual puede predecir trastornos afectivos mayores (Monteagudo et al., 2013) que tendrán repercusiones tanto en la salud del individuo como en su entorno social y familiar. En la última Encuesta Nacional de Salud, una de cada diez personas de 15 años o más declaró haber sido diagnosticada de algún problema de salud mental (Instituto Nacional de Estadística, 2018). Así, un importante número de adolescentes ha referido diversa sintomatología emocional y comportamental. En concreto el 22,6\% de una muestra de estudiantes españoles refirió nerviosismo y un 10,8\% se sintió triste o desanimado, existiendo diferencias en cuanto al género y la edad (Ortuño-Sierra, Fonseca-Pedrero, Paíno y Aritio-Solana, 2014).

El estado de ánimo negativo y la mala salud mental en la etapa adolescente se han asociado con el bullying (Mello et al., 2017; Moore et al., 2017; Singham et al., 2017), fenómeno definido como el maltrato o abuso que se da en contexto escolar entre estudiantes. La población adolescente escolarizada en España que ha sufrido bullying presenta peores puntuaciones en la escala de salud mental Strengthts and Difficulties Questionnaire (SDQ) (Garcia-Continente, Pérez-Giménez, Espelt y Nebot Adell, 2013; Mangot-Sala et al., 2018). En el Estado español ha existido una tendencia estable en los últimos años, un 4,3\% de estudiantes de 13 a 16 años han referido haber sufrido bullying (Sánchez-Queija, García-Moya y Moreno, 2017).

La adolescencia es un período durante el cual se inician numerosas conductas de riesgo como el consumo de sustancias adictivas. En España, el 38,5\% de adolescentes ha consumido tabaco, el 31,3\% cannabis y el 76,9\% alcohol alguna vez en la vida (Plan Nacional Sobre Drogas, 2016). Diferentes estudios han reportado relación entre el consumo de sustancias psicoactivas y la mala salud mental, existiendo mayor evidencia en el caso del cannabis (Fonseca-Pedrero, Ortuño-Sierra, Paino y Muñiz, 2016; Mangot-Sala et al., 2018), cuyo uso también podría aumentar el riesgo de tentativa suicida en esta población (Carvalho et al., 2018). Por lo tanto, el bullying y el consumo de sustancias podrían estar asociados con el estado anímico durante la adolescencia (Gaete et al., 2017; Garcia-Continente et al., 2013; Monteagudo et al., 2013; Moore et al., 2017). Existen estudios que han encontrado una relación entre bullying y consumo de sustancias, con problemas de ansiedad, baja autoestima, tendencias depresivas o ideación suicida en la población adolescente (Moore et al., 2017), así como con el estado de ánimo negativo (Ahonen, Nebot y Giménez, 2007).

Hasta donde conocemos, no existen estudios que analicen la posible interrelación entre el consumo de sustancias, el bullying y el estado de ánimo negativo en poblaciones pequeñas. Por este motivo, resulta de interés conocer esta re- lación en una población multicultural de menos de 10.000 habitantes, en la que conviven más de medio centenar de nacionalidades y en la que se han encontrado consumos de sustancias similares entre inmigrantes y autóctonos (Díaz Geada, Busto Miramontes y Caamaño Isorna, 2018).

De este modo, el objetivo del presente estudio es analizar la asociación entre consumo de sustancias psicoactivas, bullying y estado de ánimo negativo en estudiantes de educación secundaria.

\section{Métodos}

\section{Diseño y población del estudio}

Estudio transversal en el que se encuestaron todos los estudiantes de $2^{\circ}, 3^{\circ}$ y $4^{\circ}$ de Educación Secundaria Obligatoria (ESO) de los dos Institutos de Educación Secundaria (IES) de Burela: IES O Perdouro y Monte Castelo ( $\mathrm{n}=262)$.

\section{Recogida de datos}

La recogida de datos se llevó a cabo mediante el cuestionario FRESC (Factors de Risc en Estudiants de SeCundària), diseñado por la Agència de Salut Pública de Barcelona para conocer los comportamientos de riesgo emergentes en estudiantes de ESO. Se incluyeron dos modelos del cuestionario: uno para $2^{\circ}$ y $3^{\circ}$ de ESO (13-15 años) y otro para $4^{\circ}$ de ESO (15-16 años). Para acceder a la población de estudio, se contactó con la dirección de los centros educativos y se recabó la pertinente autorización paterna. Los datos fueron recogidos en las aulas en horario lectivo, en presencia de una docente y un miembro del equipo investigador durante diciembre del año 2015. El cuestionario fue anónimo y autocumplimentado, de forma que se garantizó en todo momento la confidencialidad de los datos.

\section{Variables}

\section{- Variable dependiente}

Estado de ánimo negativo: el estado de ánimo negativo fue medido a partir de los siguientes ítems: sentirse muy cansado/a para hacer cosas; tener problemas para quedarse dormido/a; despertarse antes de la hora; sentirse desplazado/a; sentirse triste o deprimido/a; sentirse desesperanzado/a de cara al futuro; sentirse nervioso/a en tensión y sentirse aburrido/a de las cosas. Las respuestas se ordenaron en una escala Likert de 5 puntos, desde $0=$ nunca, hasta 4 = siempre. La variable fue dicotomizada: "nunca", "casi nunca" o "a veces" tomaron el valor 0 ; mientras que "frecuentemente" y "siempre" tomaron valor 1. Las personas participantes que contestaron "frecuentemente" o "siempre" por lo menos en tres de los ítems, fueron clasificadas con estado de ánimo negativo (Ahonen et al., 2007). - Variables independientes

Como principales variables independientes se consideraron distintas variables referidas al consumo de sustancias, a la percepción de peligrosidad del consumo y al bullying. 
Respecto al consumo de sustancias, se tuvieron en cuenta:

a) Consumo de alcohol. El binge drinking se definió como haber tomado alguna vez 4 o más bebidas alcohólicas en una misma ocasión.

b) Consumo de tabaco. Se consideró haber fumado tabaco alguna vez en la vida.

c) Consumo de cannabis. Se consideró haber probado cannabis alguna vez en la vida.

La baja percepción de peligrosidad se ha asociado de forma positiva con el consumo de drogas (Ojeda, Patterson y Strathdee, 2008; Tortajada Navarro et al., 2008), por lo que se estimó la proporción de adolescentes que consideraron muy peligrosos el alcohol, tabaco y cannabis.

En el caso de la variable relacionada con el bullying, se consideraron tres ítems para la construcción de la variable: ¿Se han reído de ti o te han insultado en la escuela o de camino a ella?; ¿Te han golpeado, atacado o amenazado en la escuela o de camino a ella?; ¿A veces te marginan tus compañeros o compañeras? Las tres preguntas ofrecían cinco posibles respuestas: Nunca; una vez; dos veces; tres veces; o más de tres veces. Se consideró que los sujetos sufrían o habían sufrido bullying al contestar como mínimo a una de estas preguntas "tres veces" o "más de tres veces" o haber contestado una vez o más en los tres ítems en los últimos doce meses (Garcia-Continente et al., 2013).

Otras variables seleccionadas como potenciales variables confusoras fueron:

a) Nivel académico autorreferido. Variable medida a través de la pregunta ¿En relación a los compañeros de tu clase, en qué nivel académico dirías que te encuentras?

b) Lugar de origen. La condición de autóctono o inmigrante fue determinada en función de la nacionalidad del padre y de la madre. Fueron considerados inmigrantes los estudiantes cuyos progenitores nacieron ambos fuera de España.

c) Family Affluence Scale (FAS). Se preguntó si su familia tiene coche o furgoneta, si disponen de habitación propia, cuantos ordenadores tienen y el número de veces que han ido de vacaciones en el último año con su familia. Se sumaron las respuestas a las preguntas y se clasificaron en: FAS baja si la puntuación era de 0 a 3 , considerándose nivel socioeconómico desfavorecido; FAS media si era de 4 a 5 puntos, nivel socioeconómico medio; y FAS alta cuando la puntuación se situaba entre 6 a 7 puntos, nivel socioeconómico favorecido.

También se utilizaron como variables independientes la edady el sexo.

\section{Análisis estadístico}

Se realizó un análisis estadístico descriptivo para el global de la muestra y desglosado de acuerdo con la presencia/ausencia del estado de ánimo negativo. Se calcularon las prevalencias de estados de ánimo negativo para cada una de las variables independientes.

Para el análisis de la asociación entre las variables independientes y el estado de ánimo negativo, se estimaron modelos de regresión de Poisson univariados y multivariados con varianza robusta, obteniendo Razones de Prevalencia (RP) con sus respectivos Intervalos de Confianza al 95\% (IC95\%) (Espelt, Mari-Dell'Olmo, Penelo y Bosque-Prous, 2017). El porcentaje de valores perdidos oscilaba desde $0,42 \%$ para la variable de estados de ánimo negativo hasta 2,5\% para la variable de consumo de cannabis. Los análisis fueron realizados con el paquete estadístico STATA 15.0.

\section{Resultados}

El tamaño final de la muestra fue de 238 personas, habiendo encuestado al $91 \%$ de estudiantes de $2^{\circ}, 3^{\circ}$ y $4^{\circ}$ de ESO de Burela ( $\mathrm{n}=238)$.

En la Tabla 1 se representan las características de la muestra, según el estado anímico. La muestra estuvo compuesta por un $46,8 \%$ de chicas, un $20,7 \%$ de población inmigrante, un 47,3\% de personas de 15 o más años, un $74,3 \%$ de nivel académico medio-bajo y un 39,6\% refirió encontrarse en la FAS alta. El 10,5\% [IC95\% (7,2-15,2)] de la población encuestada presentaba estado de ánimo negativo.

La prevalencia de estado de ánimo negativo varió en función de las distintas variables independientes (Tabla 2), así en las chicas fue del 13,5\% [IC95\% (8,3-21,3)], mientras que en los chicos fue del 7,9\% [IC95\% (4,2-14,2)]. Además, fue ligeramente superior en las personas que consumían sustancias adictivas. Entre estudiantes que habían practicado binge drinking, la prevalencia de estado de ánimo negativo fue del 12,2\% [IC95\% $(5,1-26,4)]$ frente al $10,2 \%$ [IC95\% (6,6-15,3)] entre quienes no lo practicaban. Para la población adolescente que había fumado tabaco alguna vez, la prevalencia de estado anímico alcanzó el 20\% [IC95\% (8,4-40,6)] frente al 9,7\% [IC95\% (4,9-18,4)] en quienes no habían fumado tabaco. Finalmente, entre las personas que habían consumido cannabis en alguna ocasión, la prevalencia de estado de ánimo negativo ascendió al 30\% [IC95\% (9,3-64,2)] frente al 9,5\% [IC95\% (6,214,2)] en aquellas que nunca habían fumado cannabis. En lo referente al bullying, la prevalencia de este estado de ánimo también fue superior en quienes lo habían sufrido, $34,5 \%$ [IC95\% (19,3-53,3)], respecto a los que no, 7,2\% [IC95\% (4,3-11,6)].

En la Tabla 2 se presentan las Razones de Prevalencia (RP) de estado de ánimo negativo según las variables independientes, con sus correspondientes IC95\%. Así, el riesgo de tener estado de ánimo negativo fue mayor entre los estudiantes que percibían el cannabis como nada o moderadamente peligroso $\left[\mathrm{RP}_{\text {no ajustada }}=2,6\right.$; IC95\% (1,2$\left.5,5) ; \mathrm{RP}_{\text {ajustada }}=2,3 ; \operatorname{IC95\% }(1,1-4,9)\right]$, entre quienes habían 
Ainara Díaz-Geada, Albert Espelt, Marina Bosque-Prous, Núria Obradors-Rial, Ester Teixidó-Compañó, Francisco Caamaño Isorna

Tabla 1. Características sociodemográficas según los estados de ánimo en estudiantes de 13 a 16 años (Burela, 2015).

\begin{tabular}{|c|c|c|c|c|c|c|c|}
\hline & \multicolumn{2}{|c|}{ Estados de ánimo negativo } & \multicolumn{2}{|c|}{ Estados de ánimo no negativo } & \multicolumn{2}{|c|}{ Total } & \multirow[b]{2}{*}{ p-valor } \\
\hline & $n$ & $\%$ & $\mathrm{n}$ & $\%$ & $n$ & $\%$ & \\
\hline \multicolumn{8}{|l|}{ Sexo } \\
\hline Mujer & 15 & 60 & 96 & 45,3 & 111 & 46,8 & 0,136 \\
\hline Hombre & 10 & 40 & 116 & 54,7 & 126 & 53,2 & \\
\hline \multicolumn{8}{|l|}{ Nacionalidad } \\
\hline Autóctono & 20 & 80 & 168 & 79,2 & 188 & 79,3 & 0,930 \\
\hline Inmigrante & 5 & 20 & 44 & 20,8 & 49 & 20,7 & \\
\hline \multicolumn{8}{|l|}{ Edad } \\
\hline 〈15 años & 10 & 40 & 115 & 54,2 & 125 & 52,7 & 0,177 \\
\hline 〉=15 años & 15 & 60 & 97 & 45,8 & 112 & 47,3 & \\
\hline \multicolumn{8}{|l|}{ Nivel académico autorreferido } \\
\hline Bajo-Medio & 21 & 84 & 155 & 73,1 & 176 & 74,3 & 0,239 \\
\hline Alto & 4 & 16 & 57 & 26,9 & 61 & 25,7 & \\
\hline \multicolumn{8}{|l|}{ FAS } \\
\hline Baja & 4 & 16 & 42 & 20,3 & 46 & 19,8 & 0,843 \\
\hline Media & 10 & 40 & 84 & 40,6 & 94 & 40,5 & \\
\hline Alta & 11 & 44 & 81 & 39,1 & 92 & 39,6 & \\
\hline \multicolumn{8}{|l|}{ Alcohol } \\
\hline Sí & 5 & 20 & 36 & 17,0 & 41 & 17,3 & 0,706 \\
\hline No & 20 & 80 & 176 & 83,0 & 196 & 82,7 & \\
\hline \multicolumn{8}{|l|}{ Tabaco } \\
\hline Sí & 5 & 20,8 & 20 & 9,6 & 25 & 10,8 & 0,093 \\
\hline No & 19 & 79,2 & 188 & 90,4 & 207 & 89,2 & \\
\hline \multicolumn{8}{|l|}{ Cannabis } \\
\hline Sí & 3 & 12,5 & 7 & 3,4 & 10 & 4,3 & 0,038 \\
\hline No & 21 & 87,5 & 200 & 96,6 & 221 & 95,7 & \\
\hline \multicolumn{8}{|l|}{ Percepción peligro Alcohol } \\
\hline Moderado o nada peligroso & 18 & 72 & 168 & 79,3 & 186 & 78,5 & 0,404 \\
\hline Muy peligroso & 7 & 28 & 44 & 20,7 & 51 & 21,5 & \\
\hline \multicolumn{8}{|l|}{ Percepción peligro Tabaco } \\
\hline Moderado o nada peligroso & 16 & 66,7 & 133 & 64,3 & 154 & 65,3 & 0,878 \\
\hline Muy peligroso & 8 & 33,3 & 74 & 35,7 & 82 & 34,7 & \\
\hline \multicolumn{8}{|l|}{ Percepción peligro Cannabis } \\
\hline Moderado o nada peligroso & 8 & 32 & 28 & 13,4 & 36 & 15,4 & 0,015 \\
\hline Muy peligroso & 17 & 68 & 181 & 86,6 & 198 & 84,6 & \\
\hline \multicolumn{8}{|l|}{ Bullying } \\
\hline Sí & 10 & 40 & 19 & 9,0 & 29 & 12,2 & 0,000 \\
\hline No & 15 & 60 & 193 & 91,0 & 208 & 87,8 & \\
\hline
\end{tabular}

consumido esta sustancia en alguna ocasión $\left[\mathrm{RP}_{\text {no ajusta- }}\right.$ da $=3,1 ; \operatorname{IC95\% }(1,1-8,9)]$, y entre aquellos que habían sufrido bullying $\left[\mathrm{RP}_{\text {no ajustada }}=4,8 ; \operatorname{IC95\% }(2,4-9,6) ; \mathrm{RP}_{\text {ajustada }}=4,4\right.$; IC95\% (2,2-9,0)] .

En cuanto al resto de variables de consumo analizadas, no se observó ninguna asociación estadísticamente significativa, ni en el análisis bivariado ni en el multivariado; aun cuando las estimaciones puntuales sugirieron asociaciones en la misma dirección que las encontradas para el cannabis.

\section{Discusión}

El estado de ánimo negativo se ha asociado al consumo de cannabis, a la baja percepción de riesgo del consumo de 
Tabla 2. Prevalencias y Razones de Prevalencia de los estados de ánimo negativo en estudiantes de 13 a 16 años (Burela, 2015) .

\begin{tabular}{|c|c|c|c|c|c|c|c|}
\hline & \multicolumn{4}{|c|}{ Prevalencia estados de ánimo negativo } & \multirow[b]{2}{*}{ IC95\% } & \multirow[b]{2}{*}{ RPajustada } & \multirow[b]{2}{*}{ IC95\% } \\
\hline & $\mathbf{N}$ & Prevalencia (\%) & IC95\% & RPno ajustada & & & \\
\hline \multicolumn{8}{|l|}{ Sexo } \\
\hline Mujer & 111 & 13,5 & $(8,3-21,3)$ & 1 & & & \\
\hline Hombre & 126 & 7,9 & $(4,2-14,2)$ & 0,6 & $(0,3-1,2)$ & & \\
\hline \multicolumn{8}{|l|}{ Nacionalidad } \\
\hline Autóctono & 188 & 10,6 & $(6,9-15,9)$ & 1,0 & $(0,4-2,6)$ & & \\
\hline Inmigrante & 49 & 10,2 & $(4,2-22,5)$ & 1 & & & \\
\hline \multicolumn{8}{|l|}{ Edad } \\
\hline$<15$ & 115 & 8,0 & $(4,3-14,3)$ & 0,6 & $(0,3-1,3)$ & & \\
\hline$>=15$ & 97 & 13,4 & $(8,2-21,1)$ & 1 & & & \\
\hline Bajo-Medio & 155 & 11,9 & $(7,8-17,7)$ & 1,8 & $(0,6-5,1)$ & & \\
\hline Alto & 57 & 6,5 & $(2,4-16,4)$ & 1 & & & \\
\hline \multicolumn{8}{|l|}{ FAS } \\
\hline Baja & 46 & 8,7 & $(3,2-21,3)$ & 1 & & & \\
\hline Media & 94 & 10,6 & $(5,8-18,8)$ & & & & \\
\hline Alta & 92 & 11,9 & $(6,7-20,4)$ & 1,16 & $(0,7-1,9)$ & & \\
\hline \multicolumn{8}{|l|}{ Alcohol } \\
\hline Sí & 41 & 12,2 & $(5,1-26,4)$ & 1,2 & $(0,5-3,0)$ & & \\
\hline No & 196 & 10,2 & $(6,6-15,3)$ & 1 & & & \\
\hline \multicolumn{8}{|l|}{ Tabaco } \\
\hline Sí & 25 & 20 & $(8,4-40,6)$ & 2,2 & $(0,9-5,3)$ & & \\
\hline \multicolumn{8}{|l|}{ Cannabis } \\
\hline Sí & 10 & 30 & $(9,3-64,2)$ & 3,1 & $(1,1-8,9)$ & & \\
\hline No & 221 & 9,5 & $(6,2-14,2)$ & 1 & & & \\
\hline \multicolumn{8}{|l|}{ Percepción peligro Alcohol } \\
\hline Moderado o nada peligroso & 186 & 9,7 & $(6,2-14,9)$ & 0,9 & $(0,4-2,0)$ & & \\
\hline Muy peligroso & 51 & 13,7 & $(6,6-26,3)$ & 1 & & & \\
\hline \multicolumn{8}{|l|}{ Percepción peligro Tabaco } \\
\hline Moderado o nada peligroso & 154 & 10,4 & $(6,4-16,3)$ & 1,1 & $(0,5-2,3)$ & & \\
\hline Muy peligroso & 82 & 9,7 & $(4,9-18,4)$ & 1 & & & \\
\hline \multicolumn{8}{|l|}{ Percepción peligro Cannabis } \\
\hline Moderado o nada peligroso & 36 & 22,2 & $(11,4-38,9)$ & 2,6 & $(1,2-5,5)$ & 2,3 & $(1,1-4,9)$ \\
\hline Muy peligroso & 198 & 8,6 & $(5,4-13,4)$ & 1 & & 1 & \\
\hline \multicolumn{8}{|l|}{ Bullying } \\
\hline Sí & 29 & 34,5 & $(19,3-53,3)$ & 4,8 & $(2,4-9,6)$ & 4,4 & $(2,2-9,0)$ \\
\hline No & 208 & 7,2 & $(4,3-11,6)$ & 1 & & 1 & \\
\hline
\end{tabular}

esta sustancia y al hecho haber sufrido bullying. Los resultados de este estudio sugieren que el 10,5\% [(IC95\%:7,215,2)] de estudiantes de educación secundaria de Burela padece estado de ánimo negativo.

Antes de pasar a discutir los resultados hay algunas limitaciones que deberían ser comentadas. En primer lugar, el estudio tiene un diseño transversal, por lo que no permite establecer relaciones causales. Además, el consumo de alcohol, tabaco y cannabis de adolescentes fue autodeclarado. No obstante, hay evidencia de que el uso de cuestionarios autodeclarados es un método viable para medir variables de consumo de sustancias, por ejemplo el consumo de alcohol en adolescentes (Engs y Hanson, 1990). Además, la anonimidad y el formato individual del 
cuestionario podría disminuir el sesgo de deseabilidad social inherente a las encuestas. Para las variables bullying y estado de ánimo negativo se han utilizado preguntas utilizadas en distintas investigaciones (Ahonen et al., 2007; Garcia Continente, Pérez Giménez y Nebot Adell, 2010; Garcia-Continente et al., 2013; Mangot-Sala et al., 2018), sin embargo, no se conocen las propiedades psicométricas de dichas preguntas, por lo que no podemos descartar algún sesgo de clasificación. El tamaño muestral es pequeño y ha impedido disgregar por sexo, aunque cabe destacar que se ha conseguido encuestar al $91 \%$ de la población escolarizada de Burela de $2^{\circ}, 3^{\circ}$ y $4^{\circ}$ de la ESO. Es importante señalar que las poblaciones pequeñas han sido muy poco estudiadas y Burela tiene unas características migratorias que la convierten en un foco de particular interés (Oca, 2013; Pérez, Garcia-Continente y Grup col-laborador enquesta FRESC 2012, 2013).

En nuestro estudio, un $10,5 \%$ de estudiantes refirió sufrir estado de ánimo negativo, cifra que resulta consistente con los rangos referidos en nuestro entorno. Así, se han encontrado prevalencias del $16 \%$ en estudiantes catalanes de 14 y 16 años (Ahonen et al., 2007) 19\% en estudiantes de $3^{\circ}$ y $4^{\circ}$ de ESO (Monteagudo et al., 2013).

En nuestro estudio, el estado de ánimo negativo se ha asociado de forma estadísticamente significativa únicamente con el consumo de cannabis y con la percepción de peligrosidad de su consumo. Sin embargo, otros estudios sí que han encontrado relaciones estadísticamente significativas con el consumo de tabaco o el binge drinking (Julià Cano, Escapa Solanas, Marí-Klose y Marí-Klose, 2012; Martínez-Hernáez, Marí-Klose, Julià, Escapa y Marí-Klose, 2012). Esto puede deberse al tamaño muestral de nuestro estudio así como a los distintos métodos utilizados en la literatura para la medida del consumo de sustancias (Degenhardt et al., 2013; Mangot-Sala et al., 2018; Monteagudo et al., 2013; van Gastel et al., 2013). Cabe destacar que la relación causal entre el estado de ánimo negativo y el consumo de sustancias no está clara, puesto que puede darse en ambos sentidos (Merikangas et al., 1998).

La baja o nula percepción de peligrosidad del consumo de una sustancia adictiva se asocia al consumo de la misma (Ojeda et al., 2008; Tortajada Navarro et al., 2008). En este sentido, los resultados de este estudio refuerzan dicha relación en el caso del cannabis, al haber encontrado que la nula o baja percepción de peligrosidad del consumo de esta sustancia también se asocia al estado de ánimo negativo. Esto no debería menospreciarse, dado que convierte la percepción de peligrosidad en un doble factor de riesgo para la salud de los adolescentes: el consumo de cannabis junto con el estado de ánimo negativo. Esta asociación, entre la influencia en la salud mental y el consumo de cannabis en adolescentes, también fue reportada en un estudio realizado en población adolescente escolarizada de Barcelona (Mangot-Sala et al., 2018).
El estado de ánimo negativo se ha asociado a sufrir bullying. Estos resultados son consistentes con otras publicaciones (Bond, Carlin, Thomas, Rubin y Patton, 2001; Gaete et al., 2017; Garcia-Continente et al., 2013; Mangot-Sala et al., 2018; Mello et al., 2017; Monteagudo et al., 2013; Singham et al., 2017) y algún estudio longitudinal (Bond et al., 2001), que muestran que haber sufrido bullying aumenta la ansiedad y la sintomatología depresiva, o los comportamientos autodestructivos y el riesgo de suicidio en esta población. La causalidad entre el bullyingy el estado de ánimo negativo tampoco está clara, ya que el consumo de sustancias podría comportarse como una variable intermedia entre sufrir bullying y el estado de ánimo negativo (Livingston et al., 2018). Este último estudio, que monitorizó el efecto diario de haber sufrido bullying en una muestra de estudiantes norteamericanos, confirmó que el uso de tales sustancias constituía una herramienta para atenuar el estado de ánimo negativo que provocaba el bullying (Livingston et al., 2018).

\section{Conclusiones}

Existe asociación entre el estado de ánimo negativo, el consumo de cannabis y el hecho de haber sufrido bullying en la población adolescente de Burela. Nuestros resultados evidencian la necesidad de implementar medidas en el entorno escolar y social de las y los adolescentes, en pro de mejorar sus conocimientos sobre tales realidades y conseguir una detección precoz de estos comportamientos de riesgo. Así pues, sería recomendable crear intervenciones para la mejora de la salud mental durante la adolescencia que tengan en cuenta el consumo de sustancias adictivas y el hecho de haber sufrido bullying.

\section{Reconocimientos}

A todos los profesionales de los equipos de Atención Primaria que han colaborado. A B. Penabade por su continuo apoyo. A los alumnos y al personal de los Institutos de Educación Secundaria Monte Castelo y O Perdouro de Burela sin cuya ayuda y colaboración el estudio no habría sido posible. Este artículo se ha elaborado sin ninguna financiación.

\section{Conflictos de intereses}

Las autoras y autores del presente artículo declaran que no existe conflicto de intereses. Albert Espelt es editor asociado de la revista Adicciones. Sin embargo, no ha tenido ningún papel durante el proceso editorial. 


\section{Referencias}

Ahonen, E. Q., Nebot, M. y Giménez, E. (2007). Negative mood states and related factors in a sample of adolescent secondary-school students in Barcelona (Spain). Gaceta Sanitaria, 21, 43-52.

Bond, L., Carlin, J. B., Thomas, L., Rubin, K. y Patton, G. (2001). Does bullying cause emotional problems? A prospective study of young teenagers. BMJ, 323, 480-484. doi:10.1136/bmj.323.7311.480.

Carta, M. G., Fiandra, T. D., Rampazzo, L., Contu, P. y Preti, A. (2015). An Overview of International Literature on School Interventions to Promote Mental Health and Well-being in Children and Adolescents. Clinical Practice and Epidemiology in Mental Health: CP E EMH, 11(Suppl 1 M1), 16-20. doi:10.2174/1745017901511010016.

Carvalho, A. F., Stubbs, B., Vancampfort, D., Kloiber, S., Maes, M., Firth, J., ... Koyanagi, A. (2018). Cannabis use and suicide attempts among 86,254 adolescents aged 1215 years from 21 low- and middle-income countries. European Psychiatry: The Journal of the Association of European Psychiatrists, 56, 8-13. doi:10.1016/j.eurpsy.2018.10.006.

Degenhardt, L., Coffey, C., Romaniuk, H., Swift, W., Carlin, J. B., Hall, W. D. y Patton, G. C. (2013). The persistence of the association between adolescent cannabis use and common mental disorders into young adulthood. Addiction, 108, 124-133. doi:10.1111/j.13600443.2012.04015.x.

Díaz Geada, A., Busto Miramontes, A. y Caamaño Isorna, F. (2018). Alcohol, tobacco and cannabis consumption in adolescents from a multicultural population (Burela, Lugo). Adicciones, 30, 264-270. doi:10.20882/adicciones.915.

Engs, R. y Hanson, D. (1990). Gender differences in drinking patterns and problems among college students: A review of the literature. Journal of Drug and Alcohol Education, 35, 36-47.

Espelt, A., Mari-Dell'Olmo, M., Penelo, E. y Bosque-Prous, M. (2017). Applied Prevalence Ratio estimation with different Regression models: An example from a cross-national study on substance use research. Adicciones, 29, 105-112. doi:10.20882/adicciones.823.

Fonseca-Pedrero, E., Ortuño-Sierra, J., Paino, M. y Muñiz, J. (2016). Psychotic-like Experiences and Substance Use in College Students. Adicciones, 28, 144-153. doi:10.20882/adicciones.781.

Gaete, J., Tornero, B., Valenzuela, D., Rojas-Barahona, C. A., Salmivalli, C., Valenzuela, E. y Araya, R. (2017). Substance Use among Adolescents Involved in Bullying: A Cross-Sectional Multilevel Study. Frontiers in Psychology, 8, 1056. doi:10.3389/fpsyg.2017.01056.

Garcia Continente, X., Pérez Giménez, A. y Nebot Adell, M. (2010). Factores relacionados con el acoso escolar (bullying) en los adolescentes de Barcelona. Gaceta Sanitaria, 24, 103-108. doi:10.1016/j.gaceta.2009.09.017.
Garcia-Continente, X., Pérez-Giménez, A., Espelt, A. y Nebot Adell, M. (2013). Bullying among schoolchildren: Differences between victims and aggressors. Gaceta Sanitaria, 27, 350-354. doi:10.1016/j.gaceta.2012.12.012.

Instituto Nacional de Estadística. (2018). Encuesta Nacional de Salud. 2017. Recuperado de https://www. ine.es/dyngs/INEbase/es / operacion.htm?c=Estadistica_C\&cid=1254736176783\&menu=resultados\&i$\mathrm{dp}=1254735573175$.

Julià Cano, A., Escapa Solanas, S., Marí-Klose, M. y Marí-Klose, P. (2012). Psychosocial risk factors in adolescent tobacco use: negative mood-states, peer group and parenting styles. Adicciones, 24, 309-317.

Livingston, J. A., Derrick, J. L., Wang, W., Testa, M., Nickerson, A. B., Espelage, D. L. y Miller, K. E. (2018). Proximal Associations among Bullying, Mood, and Substance Use: A Daily Report Study. Journal of Child and Family Studies, 1-14. doi:10.1007/s10826-018-1109-1.

Mangot-Sala, L., Bosque-Prous, M., Bartroli, M., Teixidó-Compañó, E., Brugal, M. T. y Espelt, A. (2018). The Role of Individual and Social Mediators in the Association Between Drug Consumption and Mental Health Among Adolescents in Barcelona. International Journal of Mental Health and Addiction. doi:10.1007/s11469-018-9879-7.

Martínez-Hernáez, A., Marí-Klose, M., Julià, A., Escapa, S. y Marí-Klose, P. (2012). Heavy episodic drinking among adolescents: the association with negative mood states and family factors. Revista Española De Salud Pública, 86, 101-114. doi:10.1590/S1135-57272012000100009.

Mello, F. C. M., Silva, J. L. da, Oliveira, W. A. de, Prado, R. R. do, Malta, D. C. y Silva, M. A. I. (2017). The practice of bullying among Brazilian schoolchildren and associated factors, National School Health Survey 2015. Ciencia e Saude Coletiva, 22, 2939-2948. doi:10.1590/141381232017229.12762017.

Merikangas, K. R., Mehta, R. L., Molnar, B. E., Walters, E. E., Swendsen, J. D., Aguilar-Gaziola, S., ... Kessler, R. C. (1998). Comorbidity of substance use disorders with mood and anxiety disorders: Results of the international consortium in psychiatric epidemiology. Addictive Behaviors, 23, 893-907. doi:10.1016/S0306-4603(98)00076-8.

Monteagudo, M., Rodriguez-Blanco, T., Pueyo, M. J., Zabaleta-del-Olmo, E., Mercader, M., García, J., ... Bolíbar, B. (2013). Gender differences in negative mood states in secondary school students: health survey in Catalonia (Spain). Gaceta Sanitaria, 27, 32-39. doi:10.1016/j.gaceta.2012.01.009.

Moore, S. E., Norman, R. E., Suetani, S., Thomas, H. J., Sly, P. D. y Scott, J. G. (2017). Consequences of bullying victimization in childhood and adolescence: A systematic review and meta-analysis. World Journal of Psychiatry, 7, 60-76. doi:10.5498/wjp.v7.i1.60.

Oca, L. (2013). Caboverdianas en Burela (1978/2008): migración, relacións de xénero e intervención social. (Te- 
sis doctoral, Universidade de Santiago de Compostela). Recuperado de http://hdl.handle.net/10347/9804.

Ojeda, V. D., Patterson, T. L. y Strathdee, S. A. (2008). The influence of perceived risk to health and immigration-related characteristics on substance use among Latino and other immigrants. American Journal of Public Health, 98, 862-868. doi:10.2105/AJPH.2006.108142.

Ortuño-Sierra, J., Fonseca-Pedrero, E., Paíno, M. y Aritio-Solana, R. (2014). Prevalence of emotional and behavioral symptomatology in Spanish adolescents. Revista de Psiquiatría y Salud Mental, 7, 121-130. doi:10.1016/j. rpsm.2013.12.003.

Pérez, A., Garcia-Continente, X. y Grup collaborador enquesta FRESC 2012. (2013). Informe FRESC 2012: 25 anys d'enquestes a adolescents escolaritzats de Barcelona. Barcelona: Agència de Salut Pública de Barcelona.

Plan Nacional Sobre Drogas. (2016). Encuesta sobre uso de drogas en enseñanzas secundarias en España (ESTUDES), 1994-2014. Ministerio de Sanidad Servicios Sociales e Igualdad.

Sánchez-Queija, I., García-Moya, I. y Moreno, C. (2017). Trend analysis of bullying victimization prevalence in spanish adolescent youth at school. Journal of School Health, 87, 457-464. doi:10.1111/josh.12513.

Singham, T., Viding, E., Schoeler, T., Arseneault, L., Ronald, A., Cecil, C. M., ... Pingault, J.-B. (2017). Concurrent and Longitudinal Contribution of Exposure to Bullying in Childhood to Mental Health: The Role of Vulnerability and Resilience. JAMA Psychiatry, 74, 11121119. doi:10.1001/jamapsychiatry.2017.2678.

Tortajada Navarro, S., Valderrama Zurián, J. C., Castellano Gómez, M., Llorens Aleixandre, N., Agulló Calatayud, V., Herzog, B. y Aleixandre Benavent, R. (2008). Drug consumption and perception among Latin American immigrants. Psicothema, 20, 403-407.

van Gastel, W. A., Tempelaar, W., Bun, C., Schubart, C. D., Kahn, R. S., Plevier, C. y Boks, M. P. M. (2013). Cannabis use as an indicator of risk for mental health problems in adolescents: a population-based study at secondary schools. Psychological Medicine, 43, 1849-1856. doi:10.1017/S0033291712002723. 